\title{
The Dynamic Characteristic Research Method of Cryogenic Pneumatic Valve Based on Principal Component Analysis
}

\author{
Yongtao Qin*, Li Guo, Yongbin Li, and Liang Li \\ The $165^{\text {th }}$ Research Institute of The Sixth Academy of CASC, Xi'an, China \\ ausqinyt@126.com
}

\begin{abstract}
Keywords: Dynamic characteristic, Cryogenic pneumatic valve, Principal component analysis Abstract. To conduct quantitative analysis on the dynamic characteristics of cryogenic pneumatic valve to satisfy reliability demand, the time characteristics of cryogenic pneumatic valve were analyzed by means of the operation principle of cryogenic pneumatic valve, and dynamic characteristics model of cryogenic pneumatic valve was established by velocity dynamics theory. Based on this analysis, the key influencing factor can be defined and nonlinear quantitative analysis between dynamic characteristics and influencing factors can be completed of cryogenic pneumatic valve by principal component analysis (PCA). Finally, effectiveness of the proposed method was verified by a case study. It has shown the proposed method can improve response time and reliability of cryogenic pneumatic valve, and can provide a support for performance evaluation to rocket engine.
\end{abstract}

\section{Introduction}

The normal operation and shutdown of liquid oxygen/kerosene rocket engine was decided by reasonable starting, transferring working condition, and shutdown time order control., so that the dynamic characteristics of cryogenic pneumatic valve is a key of propellant supply [1]. But dynamic characteristics of cryogenic pneumatic valve are influenced by many factors, such as temperature, flow, structure, environment, and so on, and these factors are associated and restriction. In addition, there are nonlinear factors come from external drive signal and interior complexity structure. As result, there often is time delay problem of dynamic characteristics of cryogenic pneumatic valve in liquid oxygen/kerosene rocket engine operation or test process [2-3].

Consequently, there is a great gap between theory analyzing result and practical dynamic characteristics of cryogenic pneumatic valve, for example flow resistance error, valve core displacement error, interval flow error. When dynamic characteristics is analyzed in cryogenic system (such as liquefied natural gas, liquid hydrogen, liquid-oxygen, liquid nitrogen, and so on), there may appear a pressure liberation or flow fluctuate, and may induce the acute vibration of cryogenic system. Finally, cryogenic system will lead to pipeline leakage, blowout, malfunction, equipment breakdown, and even personnel life risk.

There were some researches of dynamic responding characteristic analyzing aspects of cryogenic valve [4-6]. However, few studies have been devoted to dynamic responding characteristic quantitative analysis of cryogenic pneumatic valve. Furthermore, few researches have investigated in dynamic responding characteristic non-linear analyzing of cryogenic valve by principal component analysis (PCA). For highly increase the start-up and ending velocity of cryogenic valve, dynamic responding characteristic of cryogenic pneumatic valve should be researched to comprehensive estimate the influencing factors of cryogenic pneumatic valve dynamic characteristics, and to conduct quantitative analysis on the dynamic characteristics of cryogenic pneumatic valve to satisfy reliability demand. This research will adapt to the development of cryogenic system, will satisfy reliability and security need of low temperature research field.

For solving delay problem of dynamic characteristics of cryogenic pneumatic valve, and conducting quantitative analysis on the dynamic characteristics of cryogenic pneumatic valve to satisfy reliability demand, the time characteristics of cryogenic pneumatic valve were analyzed by means of the operation principle of cryogenic pneumatic valve, and dynamic characteristics model of cryogenic pneumatic valve was established by velocity dynamics theory. Based on this analysis, the 
key influencing factor can be defined and nonlinear quantitative analysis between dynamic characteristics and influencing factors can be completed of cryogenic pneumatic valve by principal component analysis (PCA). Finally, effectiveness of the proposed method was verified by a case study. It has shown the proposed method can improve response time and reliability of cryogenic pneumatic valve, and can provide a support for performance evaluation to rocket engine.

\section{Time Characteristics of Cryogenic Pneumatic Valve}

An operation principle of cryogenic valve is shown in Figure 1. When electro-valve is electrified, electro-valve spool is opened and manipulation air is put in air cylinder. The pressure of manipulation air will get over the rigidity of spring, the friction force of air-proof, the action force of medium, and so on. Afterwards, the piston is drove by pressure of manipulation air to drive the movement of valve spool. Finally, this cryogenic valve is opened.

The acting force of spool valve includes the force of operation gas, the force of stable fluid flow, the friction force of gap, and the force of spring operation. The time characteristics math model of cryogenic pneumatic valve is shown as:

$$
M \frac{d^{2} x}{d t^{2}}=F_{k}-F_{i}-F_{f}-F_{t}-M g
$$

When operation gas is inlet in air cylinder, piston surface is pressed by force of operation gas. This force's direct is: the positive direct is above piston (starting direct), and the negative direct is under piston (closing direct). The force of operation gas $F_{k}$ is determined by:

$$
F_{k}=\left(P_{a}-P_{b}\right) A_{h}-k_{x} x_{0}
$$

Where, $A_{h}$ is the piston surface pressed by operation gas; $P_{a}$ is operation pressure of inlet; $P_{b}$ is operation pressure of environment.

Where $M$ is the quality of valve spool, $x$ is the movement of valve spool, $F_{k}$ is the force of operation gas, $F_{i}$ is the force of stable fluid flow, $F_{j}$ is the friction force of gap, and $F_{g}$ is the force of spring operation.

Where $M$ is the quality of valve spool, $x$ is the movement of valve spool, $F_{k}$ is the force of operation gas, $F_{i}$ is the force of stable fluid flow, $F_{j}$ is the friction force of gap, and $F_{g}$ is the force of spring operation.

The dynamic responding non-linear transferring function of cryogenic valve is shown as following:

$$
Y=\frac{\left(P_{a}-P_{b}\right) A_{h}-P_{i n} A_{a}-M g-F_{f}-k x_{0}}{M s^{3}+K_{s} s}
$$

Where, $K_{s}$ is the rigidity of valve spool spring.

\section{Dynamic Characteristics Analysis of Cryogenic Pneumatic Valve}

Principal component analysis (PCA) is a statistical procedure that uses an orthogonal transformation to convert a set of observations of possibly correlated variables into a set of values of linearly uncorrelated variables called principal components. PCA is sensitive to the relative scaling of the original variables [7-8].

The dynamic characteristics influencing factors of cryogenic pneumatic valve is analyzed by PCA:

$$
F=t_{1} p_{1}+t_{2} p_{2}+t_{3} p_{3}+\ldots . t_{n} p_{n}+E
$$

Where, $t_{n}$ is main component of system, and also is score vector. It can acquire the relationship among dynamic characteristics influencing factors of cryogenic pneumatic valve. $p_{n}$ is the characteristics vector of main component, and also is load vector. It also can acquire the relationship 
among dynamic characteristics influencing factors of cryogenic pneumatic valve. $E$ is residual matrix, and it can acquire error information of cryogenic pneumatic valve.

The contribution rate of main component is the contribution characteristic value proportion of all characteristic value. The contribution rate can analyze the number scale of resource to determine the influence degree of influence factors in dynamic characteristics influencing factors of cryogenic pneumatic valve. Finally, the key influence factor can be determined by different contribution rates. When main component number is set as $j, j=1,2 、 \cdots, n$, the contribution rate of main component in dynamic characteristics of cryogenic pneumatic valve can be described as:

$$
a_{i}=\lambda_{i} / \sum_{i=1}^{n} \lambda_{i}
$$

Where, $a_{i}$ is the $i$ th contribution rate of main component; $\lambda_{i}$ is the $i$ th characteristic value of contribution rate.

Based on dynamic characteristics analysis of cryogenic pneumatic valve by PCA, the key influence factor determine process in dynamic characteristics analysis of cryogenic pneumatic valve is defined.

\section{Application}

Taking a cryogenic valve as sample, the dynamic characteristic of cryogenic pneumatic valve based on PCA is research. The valve spool force equilibrium equation of cryogenic pneumatic valve is shown as:

$$
K_{1}\left(P_{a}-P_{b}\right) A_{h}-P_{i n} A_{a}-M g-F_{f_{-}} k_{x} x_{0}=85.79-4.56-3.34-0.053-8.72=69.12 \mathrm{kN}
$$

Based on main parameters and calculation result of dynamics, dynamic characteristic model of this cryogenic pneumatic valve is established as:

$$
5.41 \frac{d^{2} x}{d t^{2}}=69117-218000 x
$$

Because the impel signal often is phase step signal in valve operation process, dynamic responding of this cryogenic pneumatic valve is described as Figure 1. Based on the phase step responding, the responding time is $78 \mathrm{~ms}$ when starting movement length of valve spool is $25 \mathrm{~mm}$. The true measurement of responding time in this cryogenic pneumatic valve is recorded as Table 1 . This true measurement of responding time includes electro-valve spool responding time. The mean responding time is $85.6 \mathrm{~ms}$ when electro-valve spool responding time is eliminated. As result, the phase step responding, the responding time is approximate to the true measurement of responding time in this cryogenic pneumatic valve.

Table 1. The true measurement of responding time in this cryogenic pneumatic valve

\begin{tabular}{|c|c|c|c|c|c|}
\hline Number & Open time $(s)$ & End time $(s)$ & Number & Open time $(s)$ & End time $(s)$ \\
\hline 60- $\mathrm{xxx}$ & 0.116 & 0.501 & $60-\mathrm{xxx} \mathrm{A}$ & 0.162 & 0.458 \\
\hline 60- $\mathrm{xxx} \mathrm{A}$ & 0.128 & 0.491 & $60-\mathrm{xxx}$ & 0.108 & 0.518 \\
\hline $60-\mathrm{xxx}$ & 0.132 & 0.481 & $60-\mathrm{xxx} \mathrm{A}$ & 0.114 & 0.346 \\
\hline
\end{tabular}

Based on dynamic characteristic model of this cryogenic pneumatic valve, the main influencing factors include spring rigidity $X_{1}$, operation gas pressure $X_{2}$, medium pressure $X_{3}$, spring pre-compress length $X_{4}$, gas cylinder volume $X_{5}$, friction force $X_{6}$. The dynamic characteristic main component of this cryogenic pneumatic valve based on PCA is represent as:

$$
F_{1}=214.22 X_{1}-13.69 X_{2}-5.14 X_{3}+2.89 X_{4}-0.7 X_{5}-0.025 X_{6}
$$

The contribution rate distribution of main component of this cryogenic pneumatic valve is expressed as Figure 2. Based on Figure 3, spring rigidity $X_{1}$ is the maximum contribution rate in all main components in this cryogenic pneumatic valve. $X_{1}$ is key influencing factor. The opening time is shorter and valve spool movement is faster when spring rigidity $X_{1}$ is smaller in a regular spring 
pre-compress value. The spring rigidity is opposite to valve spool movement by means of dynamics theory. Consequence, there is a balance between spring rigidity and spring pre-compress length and need find a optimal value in cryogenic pneumatic valve design and apply process.

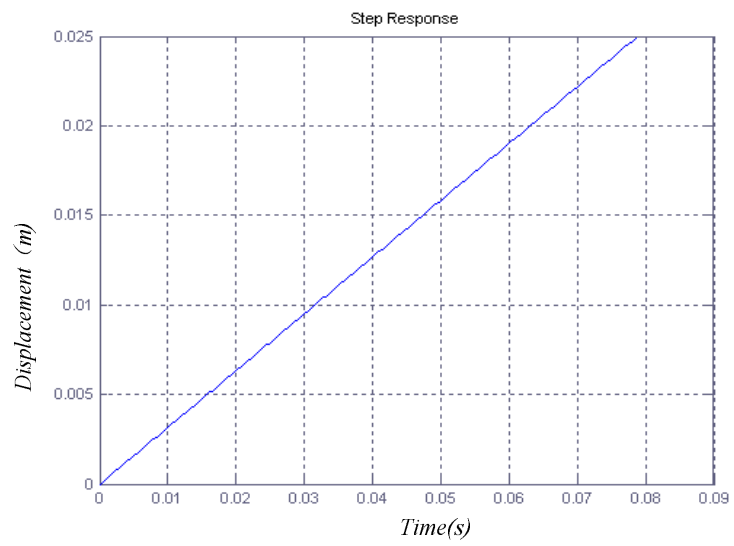

Fig. 1. The dynamic responding of this cryogenic pneumatic valve

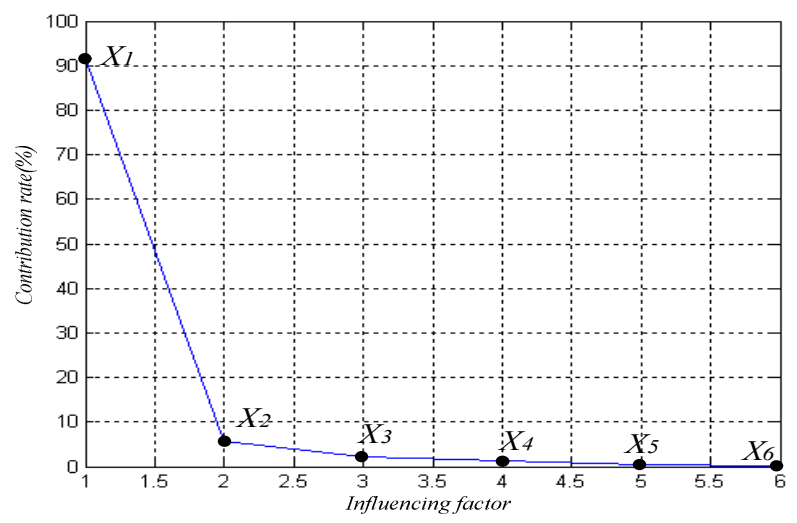

Fig. 2 The contribution rate distribution of main component in this cryogenic pneumatic valve

\section{Conclusion}

In this paper, the time characteristics of cryogenic pneumatic valve were analyzed by means of the operation principle of cryogenic pneumatic valve, and dynamic characteristics model of cryogenic pneumatic valve was established by velocity dynamics theory for solving delay problem of dynamic characteristics of cryogenic pneumatic valve. Based on this analysis, the key influencing factor can be defined and nonlinear quantitative analysis between dynamic characteristics and influencing factors can be completed of cryogenic pneumatic valve by principal component analysis (PCA). Finally, effectiveness of the proposed method was verified by a case study. It has shown the proposed method can improve response time and reliability of cryogenic pneumatic valve, and can provide an approach to rationally and exactly evaluating reliability and security of cryogenic pneumatic valve.

\section{References}

[1] Ning Zhang, Qiang Li, Qing Li, ZhongJun Hu, Kang Hu, Seat tightness of pneumatic cryogenic control valve, Science China Technological Sciences.56 (2013) 2066-2069.

[2] YiDong Fang, DaoFei Li, ZhiPeng Fan, HuanXiang Xu, Lei Wang, XiaoLi Yu, Study on pneumatic-fuel hybrid system based on waste heat recovery from cooling water of internal combustion engine, Science China Technological Sciences. 56 (2013) 3070-3080.

[3] V. D. Ananev, A. A. Belyakov, A. A. Bogdzel, M. V. Bulavin, A test setup for the bead cryogenic neutron moderator of the IBR-2 reactor, Instruments and Experimental Techniques.56 (2013) 116-122.

[4] Ning Zhang, Qiang Li, Qing Li, ZhongJun Hu, Kang Hu, Seat tightness of pneumatic cryogenic control valve, Science China Technological Sciences. 8 (2013) 2066-2069.

[5] A. M. Domashenko, I. D. Blinova,Study of heat exchange during discharge of cryogenic products into water, Chemical and Petroleum Engineering. 43 (2007) 720-725.

[6] Shu-hua Wang, Hua Zhang, Jing Wang, Experimental study on the liquid slug of cryogenic slug flow in inclined tubes, Journal of Shanghai Jiaotong University. 15 (2010) 372-376.

[7]O. Ruiz, C. Vanegas, C. Cadavid, Erratum to: Ellipse-based principal component analysis for self-intersecting curve reconstruction from noisy point sets, The Visual Computer. 27 (2011) 227-279 
[8] Vijayakumar Kadappa, Atul Negi, Divide-and-Conquer Computational Approach to Principal Component Analysis, Advances in Intelligent Systems and Computing. 327 (2015) 641-649. 